\title{
A Prototype Development of Locating Device with Graphics User Interface upon Display Using Multipoints Infrared Reflection
}

\author{
Suriyong Lertkulvanich, Tanasan Rujeera, Maytee jariyavorakit, and Suwat Buranapatpot,
}

\begin{abstract}
This project aims to develop a prototype of positioning device together with graphical user interface as a device to computer interface. The processes of device development are 1) using infrared LED lamps as light sources with a wavelength of $940 \mathrm{~nm}$ : the brightest level, for pointing out the specified location. 2) Wii Remote is a location input device and image processing to send data to a computer, and 3) reflective fabrics to reflect the light waves and using black gloves to reduce unwanted ambient light. The device is controlled by the programming the device placement and the graphical user interface on the display screen. The program is developed by using the principle of infrared light reflected at various locations. The quality of the device and the graphical user interface are evaluate by experts. The result of the project is the device works as expected as using the concept of reflective glow from the source to the reflective material and then send data to process through a series of commands that are created to locate. Moreover, the developed device can be applied to various applications and projects. The assessment by experts results that the project rank in a good level and can be update for further development
\end{abstract}

Index Terms-Graphics User Interface, Multipoints Infrared Reflection, Pointing Device

\section{INTRODUCTION}

It is a commonly known that light is the starting point of vision. It is important to the visual design. If the absence of light, human will not be able see any images and Visual Art will not happen. The effects of light exposure allow humans to aware of materials such as color, line, shape, texture, shape, weight of the composition which are elements of art. The most important thing is many theories about the relationship between light and color. In term of study of color, the study consists of light rays such as Gamma Rays, X-Rays, Ultraviolet. The visible color of light such as Infrared, Microwave, Radio Frequency waves are called Electromagnetic Spectrum. Each wave has a different wavelength. Radio waves is the longest which from $1 \mathrm{~mm}$ up to several kilometers, and Gamma rays is the shortest wavelength which less than 0.1 nanometer $(1 / 10,000,000,000$ meters $)$. Human eye can perceive electromagnetic waves in a narrow range between 780-380 nano-meter (nm.), which call Spectrum (Visible Spectrum /

Manuscript received July 15, 2011; revised September 20, 2011.

Authors are from King Mongkut's University of Technology, Thonburi, Thailand (suriyong.ler@kmutt.ac.th)
Visible Light), or in short as "light". Vary visible light is caused by a different wavelength and at different frequencies [1][2][3].

Infrared is an electromagnetic wave with a wavelength of $10^{-3}-10^{-6}$ meter. It is called infrared radiation. also known as short-wave frequencies (Millimeter waves), which overlaps with the frequency band of microwave. The infrared radiation at wavelengths shorter than $10^{-4}$ meters can be sense by human. The electromagnetic frequency is the visible spectrum that travels in a straight line. It can not pass through opaque objects. The smooth surface reflects infrared light so generally used in the communications. Infrared light wavelength is shorter than the red light. Therefore, it can not be seen by the human eye, which makes this feature sensors that use infrared light. It commonly use with the principle of reflection of light and the light transmission device. In the other words, there is a emitter of light and when the light hit the object, It will reflect light back. The reflection rate is depending on the color of objects such as black which can be reflect less than white. The surfaces that are smooth, shiny can reflect light better than roughness surface.

The applied use of infrared radiation, Pyrometers, consist with Infrared (IR) ; a component of the electromagnet. The frequency of the radiation spectrum between visible light and radio waves. Electromagnetic radiation is sinusoidal in nature and frequencies of all components of a different frequency band IR - band, between 0.7 microns and 1000 microns. They are also used to measure temperature from the surfaces of object with the use of IR sensors with high-resolution detectors, to measure the temperature of the material from the surface of the workpiece, even if the IR light is invisible to the human eye. Imagine as it is the visible light, infrared behaves in a manner similar to the other beam which travels in a straight line, absorption and attenuation by the object and anything else that comes in its path. Camera Infrared works by calculating the brightness of the room or area by LED light. The infrared LED has a special feature that can be seen on the camera. It will be light from the region of the electromagnetic spectrum that allows images to be seen even though there is a less visible light. This property is not annoy to human sensor while he stay on this kind of light area. So it is often be used as light source in many area such as computer vision, image processing etc.

The knowledge of digital image processing field is based on mathematical calculations and rules of probability which according to human vision process. Then, it analyzed and expressed in the form of the choice of one technique or other techniques desperately. The choices that use for development 
are largely from human visual behavior and estimation of vision. As the result, the development of this knowledge require an understanding the basic knowledge of vision in human first and eventually more complexity for the breadth of content. Not only cover the characteristics of human vision, but also studying the specific details which related to how the images occur in the human eye. The physical limitations of human vision is a good point for studying in terms of the factors used in our work with digital images. For this reason, factors such as comparing of image rendering in human and electronic: the resolution and the ability for adaptation or changes in the visual display is not what we are interested in, but the possibility that will occur in the images 0 .

Computer Vision is one of the filed in Artificial Intelligent that relate to the theory of image simulation system. The purpose of computer vision is enable the computer ability to create models from image. Computers can be calculate from image processing and create shapes for recording or controlling via sensor and display. The processed of computer vision include receiving image (not only by camera but also heat sensor or measurement device), image processing, modeling, and understanding image perception for simulating the model [4].

Image processing is a form of signal processing which inputs are images such as photographs or images on a video tape The output of picture processing is an image, group of features, or variables related to image process. The image processing involves improvement and changing the image, data analysis of the important information, image processing techniques including the process of converting video signals into two-dimensional signal that applied to the standard techniques of signal processing. Image Processing, which usually means, including Analog Image Processing and Digital Image Processing. Digital image is a two-dimensional function of $f(x, y)$ where $x$ and $y$ are the coordinates of the image and the amplitude of $f$ at coordinates $(x, y)$ is the value of image intensity, or the gray level of the image known as Pixels. It can be count as finite number or known as Discrete Quantity. It is a result of the reduced number of bits used to store coefficients of transformation (Quantization) that will be used to convert from analog to digital image $f(x, y)$ with size $\mathrm{M}$ rows and $\mathrm{N}$ columns, and the coordinates of the origin of the image is at position $(x, y)=(0,0)$. The computer will see the image as a number in the form of a matrix[5][6][7].

Infrared Radiation (IR); wavelength $(0.78-1000 \mu \mathrm{m})$ is range between the visible light spectrum and the microwave or the Wavenumber which is $12800-10 \mathrm{~cm}^{-1}$ by Wavenumber $\left(\mathrm{cm}^{-1}\right)=1 /$ Wavelength $(\mathrm{cm})$. Another name called under red radiation or heat radiation which wavelength is in between the red light and all kinds of radio waves at a temperature -200 degrees Celsius to 4,000 degrees Celsius. Infrared Radiation range usually use in the range of 4000-670 $\mathrm{cm}$. The skin of the human can be senses the Infrared Radiation at some wavelengths. Some films using infrared radiation for photograph. Normally, living creatures radiate infrared radiation out all the time. The infrared radiation can penetrate the thick cloud better than normal light. This property is used for imaging the earth from satellites to study the conversion of forests or movement of flocks, and so infrared radiation is also used in the control system, called remote control. It is a system for controlling operation from far distance. Infrared radiation will conduct a command from the controller to the receiver. Also in the military, bringing about the infrared radiation used to control the way of weapons to the target properly. There are currently transmitting optical fiber using infrared radiation as a vector signal because the normal light signals may be easily disturbed by ambient light.

The interaction between human and machine is different from interaction with humans to humans. While various devices have been created for using in human and machine interaction in various formats. Designers have tried to create these devices that corresponds to the human instinct as much as possible. Interaction with the computer that being used with mouse and keyboard are a standard [8]. Even if there are many invented computer systems more than the past, but in most cases these systems are often expensive includes the difficulty deployment. Since the accuracy and speed of calculation processing of the computer allowing the computer to play with people of all ages, careers. The support system for specialized applications is considered as an alternative that allows users to connect to the computer and create quality work[9]. If any agency has a convenient input device and up to date. It will enhance that agency with more reliable and improves the image of impressive to others. The system will enter the data into a computer that is important and particularly interesting.

For this reason, the researchers have been developed an input with multi-point data device for use in presentation with reflecting infrared light techniques and the image processing is done by computer. The computer is calculate several Pixel then transform into Area data such as viewing patterns or texture to determine the shape or others. Moreover, to develop a new approach in response with the computer that corresponds to the human instinct more. The price is not high and easy to use. This allows the target group can be use developed device to take advantage of innovative formats presentation, innovative and more interesting. As well as ways to interact with the computer system in the future.

\section{OBJECTIVE}

To develop a prototype of located device and the graphical user interface on the display screen using the principle of various point infrared light reflected.

\section{BENEFITS}

Benefits expected to Receive the prototype allocated device and the graphic user interface on the display screen using the principle of various point infrared light reflected.

Be able to use the prototype allocated device and the graphical user interface on the display screen using the principle of various point infrared light reflected for many purpose.

The prototype allocate device and the graphical user interface on the display screen using the principle of various point infrared light reflected can be used as guidelines for 
future development.

\section{Methodology}

The requirements are designed, the $2 \mathrm{D}$ detection area, limited of visual sensor and light intensity, human interface device and also the transfer positioning data wireless communication between light sensor device and computer. We design the project into hardward and software as follow:

\section{A. Hardware}

First, Build up Infrared LED lamps to create a light source that is used for allocate which is using the LED version TSAL6400 from Vishay Semiconductors. It contains wavelength $940 \mathrm{~nm}$ and high brightness. Next the visual input device is designed to use Wii remote as a input device for pointing and image processing to send data to a computer. In term of Wii remote, qualification by its feature which is a wireless device using the Bluetooth technology [10] contact with Broadcom BCM2042 chipset. It also using standard bluetooth protocols for contacting with human. In contact with the host that directly to human is contact ed with a USB (USB HID) device. There is a single color camera with image resolution 1024 dots horizontally and 768 dots vertically. The camera set by degrees for receiving image in the 33 degree horizontal and 23 degrees vertical. The plastic sheets that be able to filter only the infrared light best in wavelengths 940 $\mathrm{nm}$ and image processing to capture the movement of objects up to 4 pieces. Las the light sources and Wii remote (sensor) are packed in the same case as shown in Fig. 1.

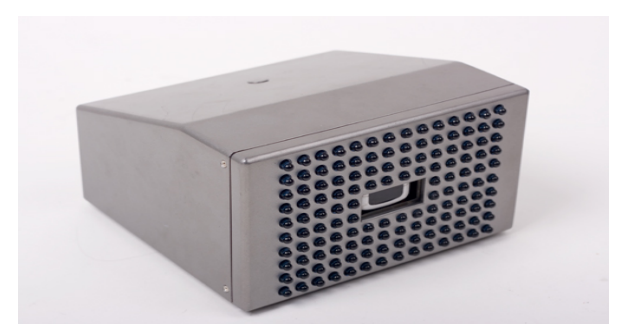

Fig. 1. A pack together of Infrared Light Sources and sensor

Another important part are the series of reflected infrared light for allocating a set of specific locations which created by using reflective fabrics following the principle of the reflection of light waves and black gloves to reduce reflection of unwanted light as shown in Fig. 2.

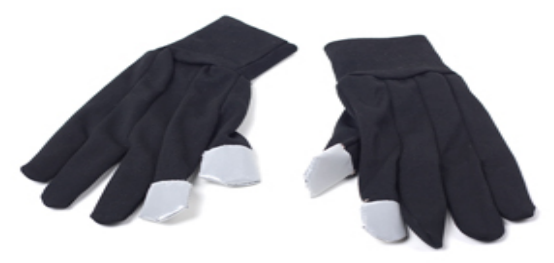

Fig. 2. Gloves with 4 points reflector that placed on fingers

\section{B. Software}

For the determination of software that need, there are two main processes, data retrieving and device to computer interface as shown in Fig. 3.

The data retrieving part process which concern with the infrared light sources and the image detection which is Wii remote, the process diagram is shown in Fig. 4. The detail of each process stage would be explained in the next items. Features of the program used to develop a prototype device for allocating the position and graphics user interface on the display screen following the principle of infrared various light reflected locations are as follows:

1. It is flexible to work with all Windows operating systems family which developed base on .NET.

2. Be able to display the location by appear on-screen display.

3. Be able to manipulate the data received from the device location that is Wii remote.

4. Be able to send data to an a variety of applications through standard communications channels.

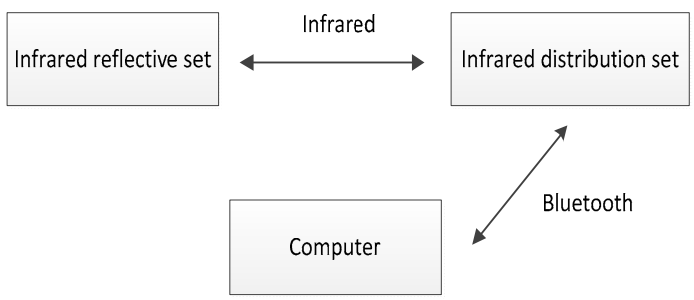

Fig. 3. The structure of the prototype allocate device and graphic user interface on the display screen using the principle of various point infrared light.

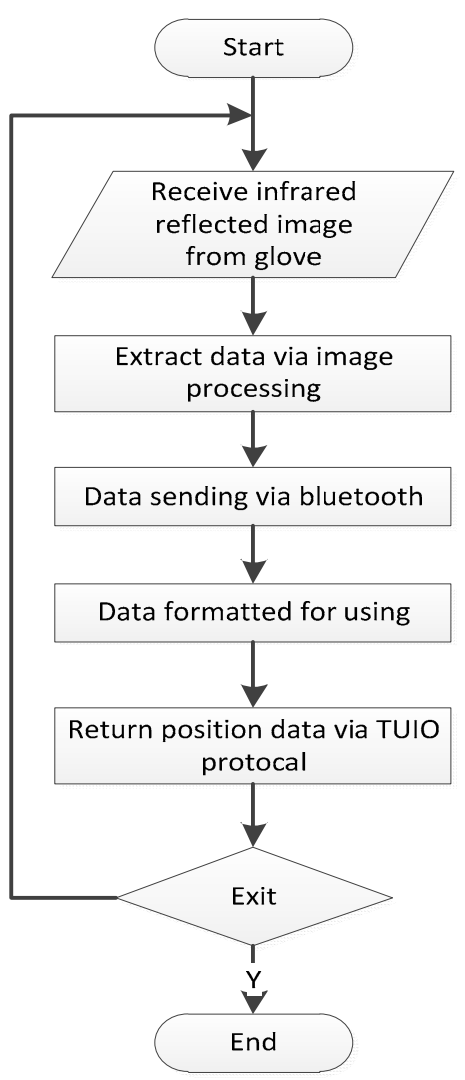

Fig. 4. The processor of prototype allocates device and graphic user interface on the display screen using the principle of various point infrared light reflected.

\section{Designing features}

For Infrared light scattering device design, in terms of light distribution, which requires enough light intensity and degree of distribution that need to be accordance with the degree of Wii remote. With the design panel the LED infrared is necessary to use the LED at the enough amount of light to get 
enough intensity to make available in approximately 2 meters. It is a distance that is appropriate for directing the movement of the arm by the user and there is a place where LED lamps on the circuit board need to form appropriate in order to make it work with the Wii remote which is a input device. Therefore, it must use TSAL6400 infrared LED lamps with power generation at 35 milliwatts per tube which provides more light intensity than general infrared LED lamps. The lamp emitting light at wavelength $940 \mathrm{~nm}$ which is not be able to see. Therefore, it will not disturb the users. The LED on the circuit board are designed to place with length ratio of $4: 3$, which corresponds to the ratio of the video image and the Wii remot. At these area, the image can be most effective. In term of Wii remote, is used as input device which is a device that can process images quickly with high resolution. It also works wirelessly so easy to work. When used with the LED circuit board, it can be developed to a devices that can emit light and input the data in a single device. For the infrared light scattering equipment, internal circuit board contains the LED and infrared Wii remote. LED infrared lamps with light distribution is designed to meet the properties of the input of the Wii remote and calculate the intensity of infrared light from the LED package reflects infrared light to design is actually works and to be associated with control of human including the principles of reflective material back to the source (Retro reflector).

The set of reflect infrared light. The set of reflects the infrared light consider by the materials to be used to reflected light from the transmitting device and to reflect infrared back to the input device directly, with minimal errors. To obtain a precise location, therefore the choice of materials requirement is the capable of reflection of light (Retro reflect). The properties of reflected light when the incident light at any direction and it will reflect back to the way. To design a set that reflects infrared light was designed to be a glove so that users can wear and use them naturally. The set of infrared light reflected in a glove contains two parts. The first part is used to reflect light back to the input device. The reflective material is a fabric capable of reflection of light for the purpose of reflection in the opposite direction to the original direction of the light. When the infrared light from the device transmitting to the reflective fabric. It will be reflected back to the video input device to be used for further processing. Another part is the gloves, which are completely black to reduce the reflection of unwanted light. Because if the users use other colors, it may cause reflection of light from infrared light scattering equipment or environment that might interfere with the reflection of light of the finger tips. This is an area where users really need to cause the reflection. The infrared equipment to generate infrared light distribution are divided to create a second step which is to create an infrared LED lamp circuit and a box to contain the LED infrared and Wii remote. The sets of reflect infrared light reflected is sewing strips of fabric attached to a black glove on the index finger and thumb on both sides.

The allocating device in this project. Users will be wearing reflective infrared gloves, which is a reflection point in the index and thumb finger tip in both hands. In operation, users will be use two fingers of each hand to the collision. It is necessary to make a cluster of points by using both hands as a criterion. The process is measured by the movement and the distance between each point. This process is inspection and to the match point as far as possible.

\section{Tracking and updating position data of each defined point}

The remote data is kept when it meets object on display. It is kept in order. In practice it can lost and unintention appear to the system. This cause the keeping data position of object on screen is not continuous and unstable. For example a moving point has a chance to lost and replace by an unintentional point. Thus there must have a process to tracking defined points and modify their position that is overlap. The process is shown in Fig. 5.

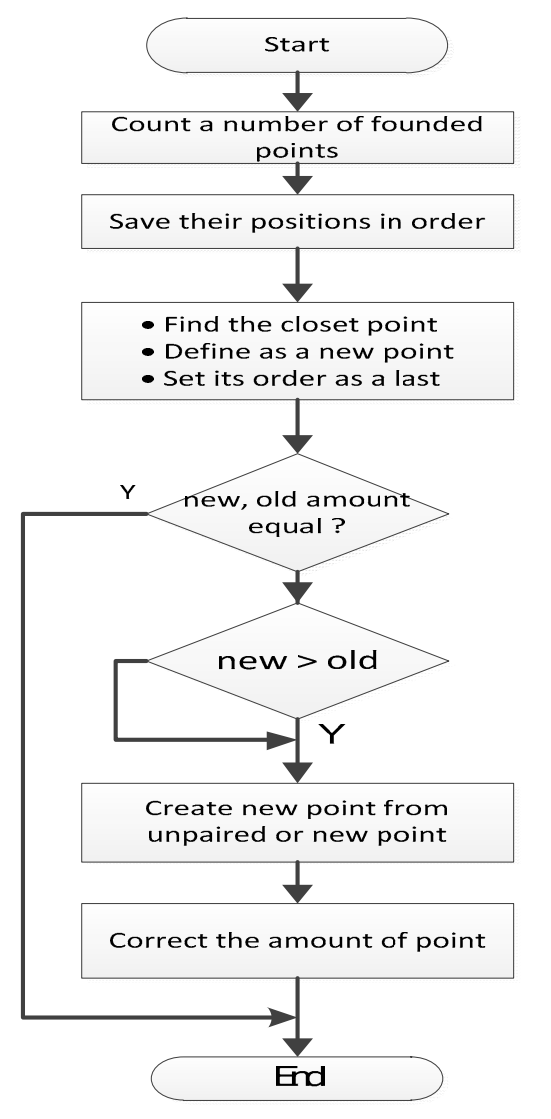

Fig. 5. Tracking and updating position data of defined point.

The process first start from counting the points found, next check the point in the current frame whether it has a number of points equal to the previous frame. If it is, a point searching process will search points which their positons mostly close to the position in previous frame. The previous points position will be encoded and replaced by current frame data to evey point. If points in both frames are not equal, a process of checking a number of point is active. For a condition of more points than previous, closing point will be used for previouse frame. The point that left will be kept as left points which will be use in next round. In case of current frame has less point than previous frame, they will be compared to every point in the previous frame and use them all. The last step of this process is modify the amount of previous frame points to equal to the current frame. This is ready to the next process. 


\section{E. Checking and paring points}

The action event of infrared reflection from user thumb and index fingers is happened when mention fingers in each hand thouching together. In order to know that which point is belong to which hand, the point must be grouped by using their distance and movement analyse process as shown in Fig. 6.

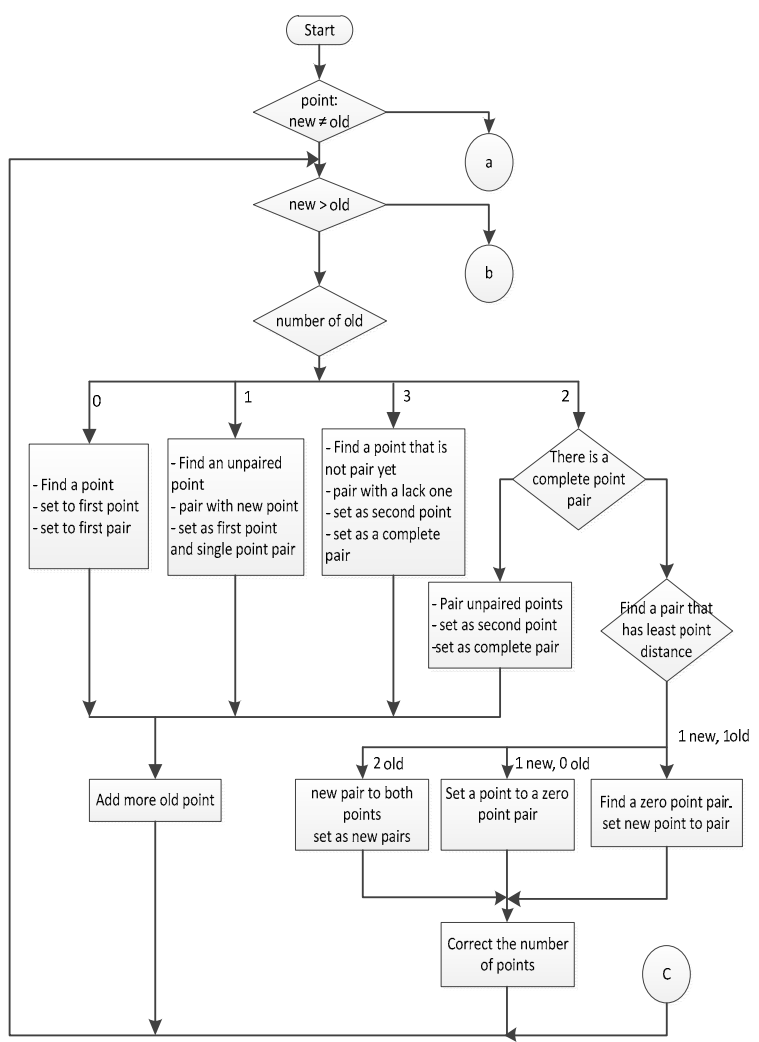

(a)

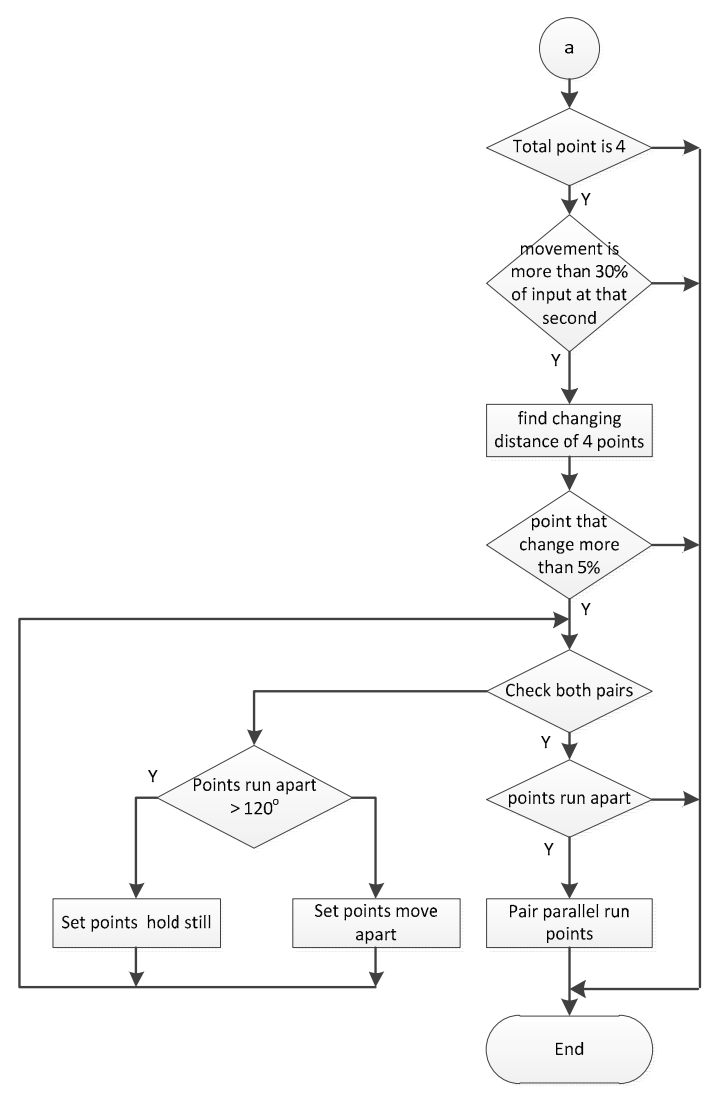

(b)

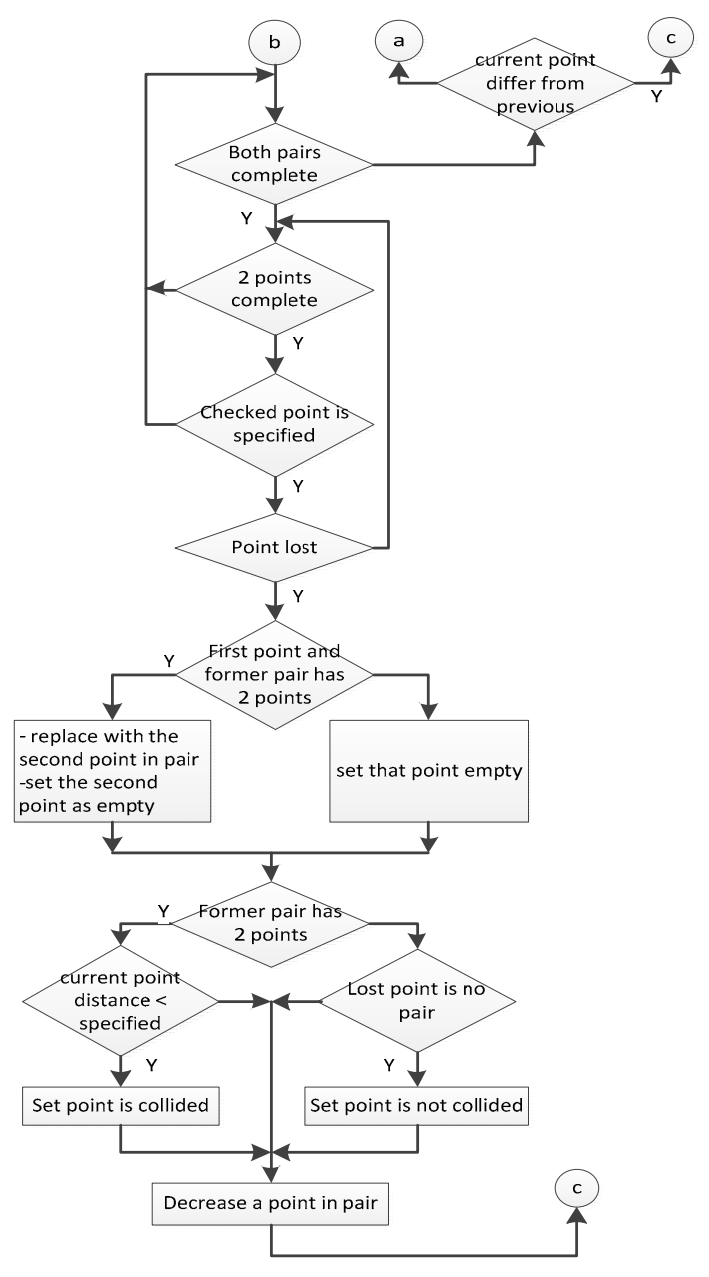

(c)

Fig. 6 .Diagram of Checking and paring points as many as posible

\section{F. Paring moving point pairs}

This case would happen when there is no sending a collided position via TUIO [13] interface. The program should send a mouse replacement data which is only one set from a point pair. Thus it must have a checking and pairing process for a point pair that moving together as shown in Fig. 7.

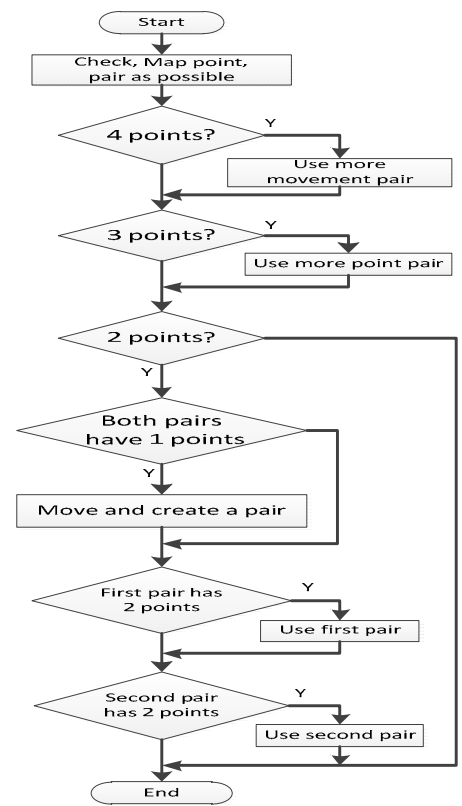

Fig. 7. Checking and pairing a moving point pair process. 
From the diagram it can be seen that it checks and pair points as many as possible. This time the concern current frame would be checked for the amount of points which is divided in 3 cases: 1, 2, 3, and 4 point on frame. For a multipoint operation it needs a point pair. The point that is single is not an interest one. Thus pair of point will be determine and create a new one if it need.

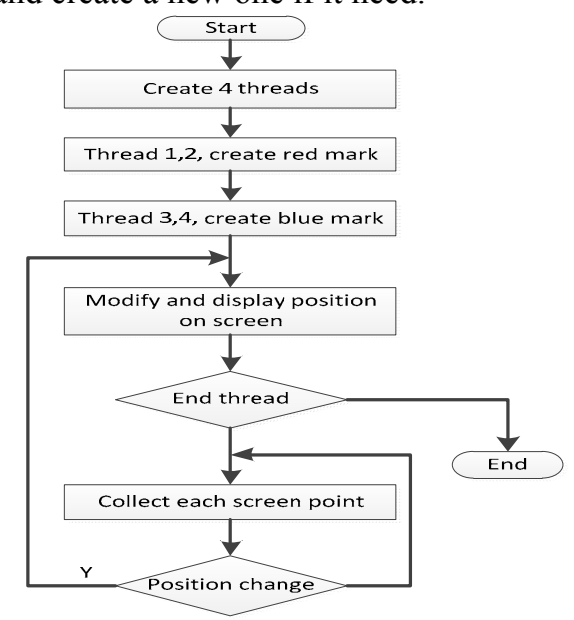

Fig. 8. Point position display on screen process

\section{G. Display points on screen}

Position from remote to processor beside placing mouse position screen it still send feedback to user. In order to continue to control, it needs to display the marks on screen. The appearance of the mark must be able to specify the point group. Process of displaying point on mark is shown in Fig. 8. It starts from creating 4 threads to support each user reflection position. The left hand uses red mark which correspond to thread 1 and 2 and blue mark for right hand correspond to thread 3 and 4 which will display on screen. The position change event will activate the process to find new position on screen. This process repeat until the end command is retrieved.

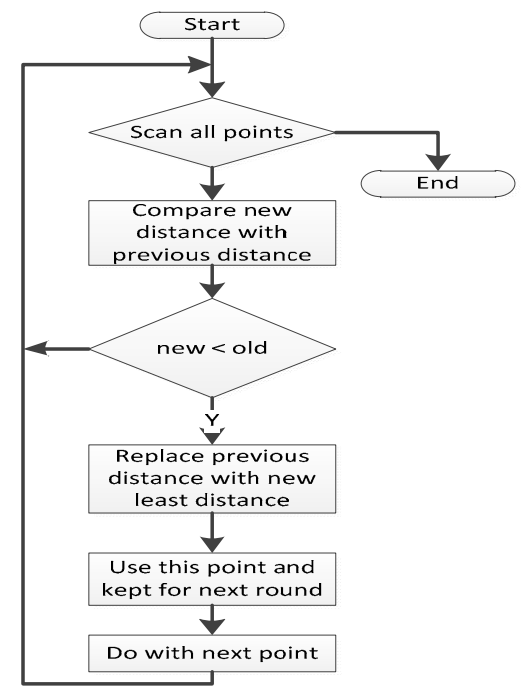

Fig. 9. Finding closet point process

\section{H. Finding a closet point}

The process of tracking and modify position data of each defined point as show in Fig. 9, requires a point from previous frame that has a closet position to the point in current frame in order to compare and analyze its position for paring. Starts from setting the closet distance value to have a maximum value. Next find the distance from reference point to the require point group, point by point. If the least distance of that point to reference is less than closet distance value, then keep its distance and point. This point will be used as a reference point for the next step also.

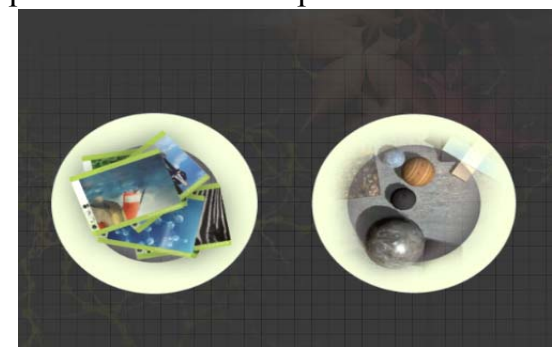

Fig. 10. Show using in API program

\section{Device functinality testing}

Some trials and experiments for position detection and matching point from the prototype device and user position and the graphic user interface [11][12] on the display screen using the principles of multi-reflected infrared light there need to know principle, which can be explained as follows:

The detect position test, there must be the reflected point occurred on the display screen which reach the requirement amounts and reflect the set of reflection package. If there is a reflection of a point, it would appear a point up on the screen as well, and display the location on the screen is associated with the actual position.

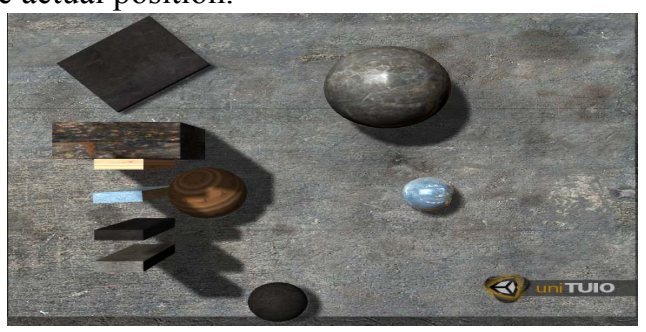

Fig. 10. Show display

The mathching point test, the four points will need to split the group into 2 groups according to both hands. The group of dots that appear on the display screen must be associated with the reflection of reflective wear on each hand side.

The last test is the utilization in various applications. testing. The positioning click were sent to various application other than prototype kits and the graphics user interface on the display screen using the principles of various point infrared light reflected can be described as follows. The click to send position result from the collision point by 2 points which must be paired together before the collision and then send out the collision point over the terms of the communication TUIO[13]. Tthe applications that support data with the same an agreement will receive the data and be processed further.

\section{CONCLUSION}

The development of a prototype allocate device and graphical user interface on the display screen using the principles of various points infrared light reflected includes infrared light which is a device that integrated circuits of 
infrared LED lights and Wii remote which is a input and image processing device. The package of infrared light reflected is a cloth glove with reflective properties that reflect light from the infrared LED circuit back to the input device for further processing. It is also including the software used to control the operation of both equipment and clothing distribution reflects infrared light together. Summarized is as follows:

The Creating the device, the building of reflective infrared light device and infrared light emitter is divided into 2 sections, the infrared light emitter is include a tube contains a circuit board of LEDs, infrared light and Wii remote and Infrared light reflector device is used materials that reflect light back (Retro reflector) to reflect infrared light. It is reflective fabric strips sewn with black gloves with the index finger and thumb on either side.

The experiments designed to test the functionality of the device. The project was divided into two parts as a result, the test to detect the position and match point. The the tool created is able to detect reflection of the user controls correctly. Both the number and relative position and correctly synchronized with the light of a hand on each side.

Procedures, first prepare the equipment and device, next wear a reflective infrared gloves which still impair to the infrared light receiver by holding the finger first. Then record the results on the display, lat extend the fingers to the fabric to reflect light back into the infrared receiver. It should be one finger by one finger, followed by the left hand thumb and left index finger, right hand thumb and right hand index finger, respectively. Recorded the result on the display.

For the result, it can show the detection of the reflected light with some accurate level, both in number and their positions relative to each point. The result is a fast and accurate in all stages of the experiment. It also can be matched accurately reflect the group's design on each side as well.

Experiments to detect the position and the matching can be done perfectly. However, in the process of development and testing, there are problems occur such as some of the points that appear on the display is flashing. The appeared in a location does not relate to their exact point. Due to environmental conditions that interfere with the reflector of the infrared light. It is causing the matching error. As well as, the complex movement led to integration positioned together. The problems that occur are fixing and providing a solution to the software. The users only need to moves hands a little, the pair will work.

Last the implementation with other applications, to test the device if it is working properly. The applications receives the data location and can be process correctly for the multitouch test with Microsoft Windows 7 operating system, launch the the Paint applications to test the drawing and also test with TUIOsmoke-0.6. The result of the test can show that the system can work well in difference overall length.

\section{DISCUSSION}

Control environmental variables to make the the device work more efficient with less error which take into account of the limitation on the distribution of light and reflect infrared light. There should be trial device often and monitor the functioning of the device to identify the performance of the device and reveal the active level if it is used for a long time, also the effectively decrease or not. These observed data can be used for adaptation of a device. To maximize efficiency, be careful with the apparel of the users. The reflection on clothes that are decorated with light reflection fabric or a shiny ring, watch buckle, etc. may interfere with the input device. The Position derived from the processing error might be occurred. In terms of materials used in the reflected light set, it needs to study the limitation of the material which uses for reflecting the infrared light. In this study, the reflective fabric may crease or scratched up on the longer use which the efficiency will decrease relatively. It cause a problem in use which should always provide backup to avoid problem. The lighting control in the using the device area should not be too dark or too bright because the amount of light was an important factor in using the developed device. If the lighting conditions in the area that does not work properly, it may be caused inaccuracy of operation of the device.

\section{REFERENCES}

[1] http://www.rmutphysics.com/charud/naturemystery/colour/colour.htm

[2] http://www.tasatec.com/index.php?lay=show\&ac=article \&Id=539165 301\&Ntype $=1$

[3] Retroreflector,[Online]Available:ttp://www.neutron.rmutphysics.com/ science-news/index.php?option $=$ com_content $\&$ task $=$ view $\& \mathrm{id}=1088 \&$ Itemid $=0$ [2010, March 5].

Image Processing, [Online], Available: http:// th.wikipedia.org/wiki [2009, January 6].

[4] Rafael C. Gonzalez and Richard E. Woods, 2002, Digital Image Processing second Edition, New Jersey: Prentice-Hall Inc, page 304-324.

[5] Computervision,[Online],Available:http ://www.cs.psu.ac.th/wiphada/ Teaching\%20Coureses/sem\%202-2547/344-

471\%20AI-ES/com_vision.ppt [2009, January 10] , page 12-143.

[6] John C. Russ., 1999, The image processing handbook, $3^{\text {rd }}$ Edition, USA: CRC Press Springer, IEEE Press, page 168-233.

[7] Randy C., 1997, A Simplified Approach to Image Processing Classical and Modern Technique in C, New Jersey, USA: Prentice Hall PTR, page 62-86.

[8] Wilbert O. G., 2007, The Essential Guide to User Interface Design : An Introduction to GUI design principles and techniques third edition, Wiley Publishing Inc, page 4 and 16.

[9] Suriyong L., Tanasan R., Maytee J., and Suwat B., The prototype of locating device with graphics user interface upon display using multipoints infrared reflection., 2011 International Conference on Economics and Business Information, IPDEDR vol.9 (2011) IACSIT Press, Bangkok Thailand, ISBN 978-981-08-8869-5.

[10] Jennifer B, Charles F. Sturman, 2001, "Bluetooth Connect Without Cables", Prentice Hall TR, United State of America, page 1-191.

[11] Soren L., 2005, User Interface Design : A software engineering perspective, Pearson, page 4.

[12] Debbie S., Caroline Jarret, Mark Woodroff, Shailey Minocha, 2005, User Interface Design and Evaluation, Morgan kaufman,page 614.

[13] TUIO document, http://www.tuio.or

Suriyong Lertkulvanich was born in October, 4, 1965, Thailand and graduate in Master of electrical engineer from Chulalongkorn university, Bangkok, Thailand since 1993. He works at the King Mongkut's University of Technology Thonburi, Faculty of Industrial Eduation and Technology in computer graphics division. He is an Assistant Professor and teach in computer graphics and computer programming and also in computer and electrical hardware in computer and information technology and multimedia division. The led him to have researches in computer graphics, computer hardware application, computer education and also computer game and mobile applications. 